\title{
Characteristics of Patients Injured in Road Traffic Accidents According to the New Injury Severity Score
}

\author{
Jung Soo Lee, MD ${ }^{1}$, Yeo Hyung Kim, MD¹, Jae Sung Yun, MD', Sang Eun Jung, MD², \\ Choong Sik Chae, $\mathrm{MD}^{3}$, Min Jae Chung, $\mathrm{MD}^{1}$
}

\begin{abstract}
${ }^{1}$ Department of Rehabilitation Medicine, Uijeongbu St. Mary's Hospital, College of Medicine, The Catholic University of Korea, Uijeongbu; ${ }^{2}$ Department of Rehabilitation Medicine, Daejeon St. Mary's Hospital, College of Medicine, The Catholic University of Korea, Daejeon; ${ }^{3}$ Department of Rehabilitation Medicine, Bucheon St. Mary's Hospital, College of Medicine, The Catholic

University of Korea, Bucheon, Korea
\end{abstract}

Objective To investigate the clinical characteristics of patients involved in road traffic accidents according to the New Injury Severity Score (NISS).

Methods In this study, medical records of 1,048 patients admitted at three hospitals located in different regions between January and December 2014 were retrospectively reviewed. Only patients who received inpatient treatments covered by automobile insurance during the period were included. Accidents were classified as pedestrian, driver, passenger, motorcycle, or bicycle; and the severity of injury was assessed by the NISS.

Results The proportion of pedestrian traffic accident (TA) was the highest, followed by driver, passenger, motorcycle and bicycle TA. The mean NISS was significantly higher in pedestrian and motorcycle TAs and lower in passenger TA. Analysis of differences in mean hospital length of stay (HLS) according to NISS injury severity revealed $4.97 \pm 4.86$ days in the minor injury group, $8.91 \pm 5.93$ days in the moderate injury group, $15.46 \pm 11.16$ days in the serious injury group, $24.73 \pm 17.03$ days in the severe injury group, and $30.86 \pm 34.03$ days in the critical injury group $(\mathrm{p}<0.05)$.

Conclusion The study results indicated that higher NISS correlated to longer HLS, fewer home discharges, and increasing mortality. Specialized hospitals for TA patient rehabilitation are necessary to reduce disabilities in TA patients.

Keywords Traffic accident, New injury severity score, Trauma, Rehabilitation

Received June 19, 2015; Accepted August 28, 2015

Corresponding author: Min Jae Chung

Department of Rehabilitation Medicine, Uijeongbu St. Mary's Hospital, College of Medicine, The Catholic University of Korea, 271 Cheonbo-ro, Uijeongbu 11765, Korea

Tel: +82-31-820-3178, Fax: +82-31-820-3177, E-mail: gigadeth04@naver.com

(c) This is an open-access article distributed under the terms of the Creative Commons Attribution Non-Commercial License (http://creativecommons.org/ licenses/by-nc/4.0) which permits unrestricted noncommercial use, distribution, and reproduction in any medium, provided the original work is properly cited. Copyright $\odot 2016$ by Korean Academy of Rehabilitation Medicine 


\section{INTRODUCTION}

Road traffic accident (RTA) is a serious public health problem and a major cause of disability worldwide. According to the World Health Organization, road traffic accidents claim 1.2 million deaths annually and cause up to 50 million non-fatal injuries [1]. In 2004, RTA ranked as the 9th leading cause of death worldwide and is expected to be the 5th by 2030 [2]. Moreover, the economic burden on society is increasing due to disabilities and deaths accompanying RTA, amounting to 518 billion US dollars per year, with the greatest losses especially in the working age population [3]. In 2014, there were a total of $20,117,955$ registered automobiles in South Korea, and in 2012, there were 1,133,145 RTA, with 5,392 deaths and $1,777,604$ injuries, thus showing the tendency of gradual increase in incidence of RTA. Compared to other Organization for Economic Co-operation and Development (OECD) member countries, of which $68.2 \%$ of the OECD average was 64.4 vehicles per 100 people, 43.9 per 100 people in South Korea owned automobiles as of 2010. However, there were 464.2 traffic accidents per 100,000 people, ranking 3rd, following the United States and Japan. In terms of number of traffic accidents, there were 105.8 traffic accidents per 10,000 vehicles, which placed South Korea in the 1st rank. Since the number of traffic accident patients is gradually increasing, identifying their clinical characteristics is important in planning rehabilitation program during acute inpatient treatment.

The Injury Severity Score (ISS), which was proposed in 1974 by Baker et al. [4], reflects deteriorating patients' conditions following trauma and is widely used as an indicator of the severity of anatomic injuries. However, since it only reflects a single injury from each body region, its clinical utility is limited. In order to address this problem, Osler et al. [5] proposed the New Injury Severity Score (NISS) in 1997; the current study used this tool to assess injury severity. This study was designed to assess the clinical characteristics of patients involved in traffic accident (TA) with NISS.

\section{MATERIALS AND METHODS}

\section{Subjects}

This study was approved by the Institutional Review Board of Uijeongbu St. Mary's Hospital. We retrospec- tively reviewed medical records of 1,048 patients admitted at three hospitals located in different regions between January and December 2014. Only patients who received inpatient treatments covered by automobile insurance during the period were included; whereas, those who were hospitalized or treated for sequela of accident were excluded. TA were defined as accidents involving personal injuries from vehicle traffic on the road.

\section{Methods}

Patients' gender, age, accident type, utilization of rehabilitative medicine or consultation, requirement for surgery, presence of multiple injuries, injury severity, hospital length of stay (HLS), Glasgow Coma Score (GCS) at admission, and type of discharge were investigated. Accidents were classified as pedestrian, driver, passenger, motorcycle, or bicycle, while discharge types included death, home discharge, university hospital, general hospital, rehabilitation hospital, convalescent hospital, and private hospital. In addition, intervening factors of rehabilitation therapy, such as presence of indwelling catheter, nasogastric tube, or endotracheal tube were further investigated.

The NISS was used to assess injury severity. To compute the NISS, each injury was classified according to body regions (head or neck, face, chest, abdominal or pelvic contents, extremities or pelvic girdle, and external) and severity, which was based on an abbreviated injury scale (1, minor; 2 , moderate; 3 , severe, not life-threatening; 4 , severe, life-threatening, survival probable; 5 , critical, survival uncertain; and 6, fatal injury). Next, regardless of body regions, the scores from the three regions in order of highest injury severity were squared and summed [6]. For example, a person with multiple injuries to the face, chest, and extremities with injury scale of graded 2, 1, and 3 , respectively, had a NISS of 14 . According to a study by Stevenson, et al. [7], injury severities in the present study were classified based on NISS score: minor (1-3 points), moderate (4-8 points), serious (9-15 points), severe (16-25 points), or critical (25-75 points).

\section{Statistical analysis}

ANOVA test was used to compare subgroups according to accident type, age, NISS, and transfer type, and Scheffe post-hoc test was also performed. Independent t-test was used to compare NISS according to the presence of the 
catheter or tube. The p-values less than 0.05 were considered statistically significant.

\section{RESULTS}

\section{General characteristics of the subjects}

The age of subjects ranged from 1 to 94 years, with a mean age of $48.75 \pm 20.64$ years; $128(12.2 \%)$ were 20 years or below, $213(20.3 \%)$ were $21-40$ years, 381 (36.4\%) were $41-60$ years, and 326 (31.1\%) were 61 years or older. The mean of NISS in 20 years or below was $13.25 \pm 13.79$, $12.91 \pm 13.70$ in $21-40$ years, $15.08 \pm 13.61$ in $41-60$ years, and 19.03 \pm 15.55 in 61 years or older. Post-hoc Scheffe test showed that the mean of NISS was significantly higher in 61 years or older group $(\mathrm{p}<0.05)$. There were 621 men $(59.3 \%)$ and 427 women $(40.7 \%)$ in the study population.

Most patients ( $\mathrm{n}=871,83.1 \%$ ) had a GCS of 15 at admission, while 26 patients $(2.5 \%)$ had a GCS of 3 . A total of 176 patients (16.8\%) had central nervous system injuries, including 172 (16.4\%) with traumatic brain injury; 15 (1.4\%) had spinal cord injuries, and 11 (1.0\%) had both. In addition, 141 patients (13.5\%) required long-term bed rest due to femur or spine fractures. In terms of the proportion of patients with major injuries, such as central nervous system injuries and femur or spine fracture, according to accident type, bicycle TA comprised $42.5 \%$, while motorcycle, driver, passenger, and pedestrian TAs made up the remaining accidents, at $38.9 \%, 20.9 \%, 26.7 \%$, and $34.7 \%$, respectively.

A total of 503 (48.0\%) patients underwent surgeries due to TA injury, and 701 (66.9\%) incurred multiple traumas. Extremities were most often injured, with 498 cases (47.5\%) followed by head and neck $(\mathrm{n}=337,32.2 \%)$, face $(\mathrm{n}=296,28.2 \%)$, chest $(\mathrm{n}=283,27.0 \%)$, and abdomen and pelvis $(\mathrm{n}=237,22.6 \%) ; 298$ patients $(28.4 \%)$ had upper or lower extremity fractures (Table 1 ).

\section{Accident types and NISS}

Pedestrian TAs were most common, with 383 (36.5\%) cases, followed by driver $(\mathrm{n}=249,23.8 \%)$; passenger $(\mathrm{n}=187,17.8 \%)$; motorcycle $(\mathrm{n}=149,14.2 \%)$; and bicycle TAs ( $\mathrm{n}=80,7.6 \%)$. The mean of NISS in pedestrian TA was $18.93 \pm 15.33,17.72 \pm 15.53$ in motorcycle TA, $15.48 \pm 14.75$ in bicycle TA, $12.99 \pm 13.63$ in driver TA, and 10.97 \pm 10.26 in passenger TA. Post-hoc Scheffe test showed that the mean of NISS was higher in pedestrian and motorcycle
TAs and significantly lower in passenger TAs, which were statistically significant $(\mathrm{p}<0.05)$ (Table 2$)$.

\section{HLS and NISS}

A total of $483(46.1 \%)$ patients were hospitalized $\leq 10$ days, 284 (27.1\%) for 11-20 days, 122 (11.6\%) for 21-30 days, 58 (5.5\%) for 31-40 days, and 101 (9.6\%) for $\geq 41$ days. Analysis of injury severity by NISS revealed 115

Table 1. Description of the study population

\begin{tabular}{|c|c|}
\hline Characteristic & Value \\
\hline Age (yr) & $48.75 \pm 20.64$ \\
\hline \multicolumn{2}{|l|}{ Gender } \\
\hline Male & $621(59.3)$ \\
\hline Female & $427(40.7)$ \\
\hline \multicolumn{2}{|l|}{ Accident type } \\
\hline Pedestrian & $383(36.5)$ \\
\hline Driver & $249(23.8)$ \\
\hline Passenger & $187(17.8)$ \\
\hline Motorcycle & $149(14.2)$ \\
\hline Bike & $80(7.6)$ \\
\hline \multicolumn{2}{|l|}{ Operation } \\
\hline Yes & $503(48.0)$ \\
\hline No & $545(52.0)$ \\
\hline \multicolumn{2}{|l|}{ CNS injury } \\
\hline TBI & $172(16.4)$ \\
\hline SCI & $15(1.4)$ \\
\hline Combined & $11(1.0)$ \\
\hline \multicolumn{2}{|l|}{ Injury region } \\
\hline Head and neck & $337(32.2)$ \\
\hline Face & $296(28.2)$ \\
\hline Chest & $283(27.0)$ \\
\hline Abdomen and pelvis & $237(22.6)$ \\
\hline Extremity & $498(47.5)$ \\
\hline \multicolumn{2}{|l|}{ Discharge status } \\
\hline Death & $41(3.9)$ \\
\hline Home discharge & $627(59.8)$ \\
\hline University hospital & $60(5.7)$ \\
\hline Rehabilitation hospital & $22(2.1)$ \\
\hline General hospital & $174(16.6)$ \\
\hline Convalescent hospital & $43(4.1)$ \\
\hline Private hospital & $71(6.8)$ \\
\hline
\end{tabular}

Values are presented as mean \pm standard deviation or number of patients per group (the percentage within each group). CNS, central nervous system; TBI, traumatic brain injury; SCI, spinal cord injury. 
Table 2. Clinical characteristics of study population according to accident type

\begin{tabular}{lccccc}
\hline & Bicycle TA & Motorcycle TA & Driver TA & Passenger TA & Pedestrian TA \\
\hline No. of patient & $80(7.6)$ & $149(14.2)$ & $249(23.8)$ & $187(17.8)$ & $383(36.5)$ \\
Age (yr) & $54.06 \pm 23.08$ & $46.32 \pm 20.56$ & $48.60 \pm 14.36$ & $47.17 \pm 21.69$ & $49.45 \pm 22.83$ \\
Hospital length of stay (day) & $21.23 \pm 43.81$ & $18.72 \pm 18.91$ & $11.96 \pm 11.78$ & $13.06 \pm 14.37$ & $22.06 \pm 20.94$ \\
NISS & $15.48 \pm 14.75^{*}$ & $17.72 \pm 15.53^{*}$ & $12.99 \pm 13.63^{*}$ & $10.97 \pm 10.26^{*}$ & $18.93 \pm 15.33^{*}$ \\
Discharge status & & & & & \\
Death & $6(7.5)$ & $8(5.4)$ & $7(2.8)$ & $1(0.5)$ & $19(5.0)$ \\
Home discharge & $46(57.5)$ & $78(52.3)$ & $169(67.9)$ & $131(70.1)$ & $221(57.7)$ \\
Transfer & $28(35.0)$ & $63(42.3)$ & $73(29.3)$ & $55(29.4)$ & $161(42.0)$ \\
\hline
\end{tabular}

Values are presented as mean \pm standard deviation or number of patients per group (the percentage within each group).

TA, traffic accident; NISS, New Injury Severity Score.

${ }^{*} \mathrm{p}<0.05$ from one-way ANOVA.

Table 3. The comparison of hospital length of stay according to New Injury Severity Score

\begin{tabular}{lccccc}
\hline & \multicolumn{5}{c}{ New Injury Severity Score } \\
\cline { 2 - 6 } & $\mathbf{1 - 3}$ (mild) & $\mathbf{4 - 8}$ (moderate) & $\mathbf{9 - 1 5}$ (serious) & $\mathbf{1 6 - 2 4}$ (severe) & $\mathbf{2 5 - 7 5}$ (critical) \\
\hline No. of patient & $115(11.0)$ & $271(25.9)$ & $273(26.0)$ & $139(13.3)$ & $250(23.9)$ \\
Hospital length of stay (day) & $4.97 \pm 4.86^{*}$ & $8.91 \pm 5.93^{*}$ & $15.46 \pm 11.16^{*}$ & $24.73 \pm 17.03^{*}$ & $30.86 \pm 34.03^{*}$ \\
\hline
\end{tabular}

Values are presented as mean \pm standard deviation or number of patients per group (the percentage within each group). ${ }^{*} \mathrm{p}<0.05$ from one-way ANOVA.

cases $(11.0 \%)$ of mild injury ( $\leq 3$ points); $271(25.9 \%)$ of moderate injury (4-8 points); $273(26.0 \%)$ of serious injury (9-15 points); 139 (13.3\%) of severe injury (16-24 points); and 250 (23.9\%) cases of critical injury (25-75 points). Analysis of differences in mean HLS according to NISS injury severity revealed $4.97 \pm 4.86$ days for patients in the minor injury group (NISS, $1-3$ ), 8.91 \pm 5.93 days in the moderate injury group (NISS, 4-8), $15.46 \pm 11.16$ days in the serious injury group (NISS, 9-15), 24.73 \pm 17.03 days in the severe injury group (NISS, 16-24), and 30.86 \pm 34.03 days in the critical injury group (NISS, 25-75), with statistically significant differences $(\mathrm{p}<0.05)$ (Table 3$)$. Post-hoc Scheffe test showed that higher NISS was significantly correlated with longer HLS $(\mathrm{p}<0.05)$.

\section{Condition at discharge and NISS}

Among study subjects, 41 patients (3.9\%) died and 7 $(0.7 \%)$ were discharged in a comatose state. A total of 713 (68.0\%) patients had independent gait without serious complication, while 287 patients (27.4\%) were discharged with required assistance in gait and activity of daily living. The mean NISS was $10.21 \pm 9.46$ in the independent gait group, $24.63 \pm 14.86$ in the group requiring assistance in gait, $48.71 \pm 14.84$ in the comatose state group, and $42.02 \pm 15.94$ in the group of patients who had died, with significant group-wise differences $(\mathrm{p}<0.05)$.

Most patients were discharged to their homes $(n=627$, $59.8 \%)$, while $60(5.7 \%)$ and $22(2.1 \%)$ patients were discharged to university and rehabilitation hospitals, respectively; in addition, $174(16.6 \%), 43(4.1 \%)$, and 71 (6.8\%) patients were discharged to general, convalescent, and private hospitals, respectively. The mean NISS was $9.78 \pm 8.67$ in the home discharge group, $22.52 \pm 15.71$ in the transfer group, and $42.02 \pm 15.94$ in the group of patients who had died. Post-hoc Scheffe test showed that the mean of NISS was significantly higher in the group of patients who had died and significantly lower in the home discharge group $(\mathrm{p}<0.05)$.

\section{Rehabilitation therapy}

Among patients in this study, 137 (13.1\%) underwent rehabilitation consultation, including $13(1.2 \%)$ who were transferred to the department of rehabilitation medicine for intensive treatment. Indwelling catheters were inserted in 342 patients (32.6\%); while 74 patients (7.1\%) received a nasogastric tube and $92(8.8 \%)$ underwent in- 
Table 4. The comparison of New Injury Severity Score according to the presence of catheter or tube

\begin{tabular}{lccc}
\hline & $\begin{array}{c}\text { Indwelling } \\
\text { catheter }\end{array}$ & $\begin{array}{c}\text { Nasogastric } \\
\text { tube }\end{array}$ & $\begin{array}{c}\text { Endotracheal } \\
\text { tube }\end{array}$ \\
\hline Yes & $28.60 \pm 16.09$ & $42.04 \pm 15.95$ & $41.27 \pm 16.11$ \\
No & $9.40 \pm 8.04$ & $13.66 \pm 12.20$ & $13.20 \pm 11.63$ \\
p-value & $<0.05$ & $<0.05$ & $<0.05$ \\
\hline
\end{tabular}

Values are presented as mean \pm standard deviation.

tubation. NISS according to the presence of the catheter or tube in each group showed a statistically significant difference $(\mathrm{p}<0.05)$ (Table 4$)$.

\section{DISCUSSION}

The ISS has been considered as the gold standard of assessing injury severity; however, it possesses weakness in the evaluation of multiple skeletal injuries [8]. In a person with multiple fractures, the ISS would be calculated based on the most severely injured site, and the total severity may be overlooked by ignoring other significant fractures [9]. In order to address this problem, Osler et al. [5] proposed the NISS in 1997. The NISS considers the three most severe injuries, regardless of body region. According to Lavoie et al. [6,8], NISS is not only a more correct predictor of in-hospital mortality than the ISS, but also helpful in forecasting intensive care unit admission and HLS. Moreover, according to Sutherland et al. [9], NISS is helpful in predicting functional outcomes such as mobility, house work or self- care in patients of musculoskeletal trauma. The results of the present study indicated that higher NISS was correlated to longer HLS, fewer home discharges, and increasing mortality. Among accident types, NISS was high in pedestrian and motorcycle TA, which consequently showed higher rates of poor prognosis.

Injuries from TAs result in tremendous personal and social losses. They can diminish quality of life and can lead to functional and occupational activity impairments, as well as psychological problems in road traffic injured patients $[10,11]$. In South Korea, there were a total of $1,133,145$ cases, 5,392 deaths, and 1,777,604 people injured from TAs in 2012. As compared to statistics data of the previous year, the number of both, total TA and subsequent death, were increased $(26.3 \%$ and $3.1 \%$ increase, respectively). Hence, the need for systematic rehabilita- tion therapy to reduce complications is also anticipated to increase.

Receiving appropriate rehabilitation therapy after injury is important for improving functional outcomes. Brooke et al. [12] examined patients with fractures from TAs and reported positive effects on pain and return to work in the patient group that underwent early intervention through rehabilitation consultation. Despite the fact that many TA patients require rehabilitation to achieve improved prognosis, only $13.1 \%$ of patients in the present study received evaluation and management by the department of rehabilitative medicine during their hospitalization. There were several possible reasons for this fact. First, injuries that were restricted to face, rib or minor injuries such as laceration were difficult to be considered as indication for rehabilitation. Moreover, some patients that were in life-threatening conditions, such as internal organ damage or septic condition, could not endure the rehabilitation program due to poor general condition. Finally, discharge from the hospital was mandated in some patients due to limited duration of hospital stay. A number of other factors besides rehabilitation therapy can also influence the prognosis of TA patients. Fort et al. [13] reported that severe lower-limb lesions, severe thoracic lesions, and cranial trauma significantly delayed patients' return to work.

Improving functional outcome and preventing disability in TA patients are important from a cost-savings perspective, not only for the patients, but also for the society as a whole. Prolonged time of returning to workplace and loss of work capacity due to TA-related complications reduce quality of life of the patients and family, and can lead to inefficient distribution of limited medical resources. Currently, it is difficult to provide sufficient functional assessment and early rehabilitation therapy to patients with trauma; considering the present medical insurance system, treatment is even more difficult for patients with fractures or internal organ damage since they often require long-term HLS. To reduce patients' disabilities and improve prognosis, management of socioeconomic and psychological factors during the subacute stage is needed; however, as mentioned earlier, adequate care is difficult due to limit in HLS and other issues. Therefore, sufficient hospitals that can provide specialized treatment to TA patients are required, in order to improve the quality of life for patients and their families and reduce 
the social loss of patients.

The present study has several limitations. Functional and psychological evaluations, which are expected to influence patients' prognosis, were not sufficiently conducted; furthermore, since no follow-up observations were made due to the retrospective nature of the study design, data on complications, such as pain, that can occur after discharge were unavailable. In addition, future investigations on the association between return to work and quality of life would be meaningful.

In the present study, the medical records of automobile insurance patients who were admitted to three hospitals located in different regions in 2014 were investigated to identify clinical trends in TA patients. The prognostic factors for patients included accident type and initial injury severity, while higher NISS showed a pattern of prolonged treatment duration and increased mortality. Although comprehensive rehabilitation therapy is needed to reduce disabilities in TA patients, it is currently difficult to adequately undertake such tasks, and specialized hospitals for TA patient rehabilitation are necessary. The results of the present study may inform development of adequate rehabilitation therapy for TA patients and can be used as fundamental data for more broad-based studies in the future.

\section{CONFLICT OF INTEREST}

No potential conflict of interest relevant to this article was reported.

\section{REFERENCES}

1. Toroyan T. Global status report on road safety. Inj Prev 2009;15:286.

2. World Health Organization. World health statistics 2008. Geneva: World Health Organization; 2008.

3. Ameratunga S, Hijar M, Norton R. Road-traffic injuries: confronting disparities to address a global-health problem. Lancet 2006;367:1533-40.
4. Baker SP, O'Neill B, Haddon W Jr, Long WB. The injury severity score: a method for describing patients with multiple injuries and evaluating emergency care. J Trauma 1974;14:187-96.

5. Osler T, Baker SP, Long W. A modification of the injury severity score that both improves accuracy and simplifies scoring. J Trauma 1997;43:922-6.

6. Lavoie A, Moore L, LeSage N, Liberman M, Sampalis JS. The New Injury Severity Score: a more accurate predictor of in-hospital mortality than the Injury Severity Score. J Trauma 2004;56:1312-20.

7. Stevenson M, Segui-Gomez M, Lescohier I, Di Scala C, McDonald-Smith G. An overview of the injury severity score and the new injury severity score. Inj Prev 2001;7:10-3.

8. Lavoie A, Moore L, LeSage N, Liberman M, Sampalis JS. The Injury Severity Score or the New Injury Severity Score for predicting intensive care unit admission and hospital length of stay? Injury 2005;36:477-83.

9. Sutherland AG, Johnston AT, Hutchison JD. The new injury severity score: better prediction of functional recovery after musculoskeletal injury. Value Health 2006;9:24-7.

10. Hours M, Chossegros L, Charnay P, Tardy H, Nhac-Vu HT, Boisson D, et al. Outcomes one year after a road accident: results from the ESPARR cohort. Accid Anal Prev 2013;50:92-102.

11. Kenardy J, Heron-Delaney M, Warren J, Brown EA. Effect of mental health on long-term disability after a road traffic crash: results from the UQ SuPPORT study. Arch Phys Med Rehabil 2015;96:410-7.

12. Brooke KJ, Faux SG, Wilson SF, Liauw W, Bowman M, Klein L. Outcomes of motor vehicle crashes with fracture: a pilot study of early rehabilitation interventions. J Rehabil Med 2014;46:335-40.

13. Fort E, Bouffard E, Charnay P, Bernard M, Boisson D, Laumon B, et al. Return to work following road accidents: factors associated with late work resumption. J Rehabil Med 2011;43:283-91. 